\title{
Protective Effects and Mechanisms of Babao Dan Capsules on ANIT-Induced Intrahepatic Cholestasis Liver Disease in Mice
}

Hua Mao ( $1109663087 @ q q . c o m$ )

Zhujiang Hospital https://orcid.org/0000-0003-2414-4896

Guowei Liu

Zhujiang Hospital

Liyun Huang

Zhujiang Hospital

Shiyi Xie

Zhujiang Hospital

Min Lu

Zhujiang Hospital

Chuan Liu

Zhujiang Hospital

Research

Keywords: Babaodan, cholestasis, liver fibrosis, mice

Posted Date: June 8th, 2021

DOI: https://doi.org/10.21203/rs.3.rs-557944/v1

License: (1) This work is licensed under a Creative Commons Attribution 4.0 International License.

Read Full License 


\section{Abstract \\ Background}

Babao Dan(BBD), as a traditional Chinese medicine, is widely used in a variety of diseases, especially chronic liver diseases. The aim of this study is to observe the effects and possible mechanisms of Babao Dan(BBD) Capsules on a-Naphthyl isothiocyanate (ANIT) - induced intrahepatic cholestasis liver disease in mice.

\section{Methods}

Fifteen mice were randomly divided into three groups: control group, model group, and treatment group, with five in each group. Except for the mice in the control group, the mice in the other two groups were gavaged with a $1 \%$ a-Naphthyl isothiocyanate (ANIT) olive oil solution, and cholestasis models were established at a dose of $100 \mathrm{mg} / \mathrm{Kg}$. Meanwhile, at the same time point, the control group was gavaged with the same amount of olive oil. After modeling, the control group, the model group and the treatment group were gavaged with the same amount of saline, saline and BBD saline suspension once daily for 10 days, respectively. On the 10th day, we took the blood preparation and liver samples, then we detected serum levels of liver function indexes(TBA, TBil, DBil, ALT, AST, ALP, GGT), liver fibrosis indexes(HA, LN,

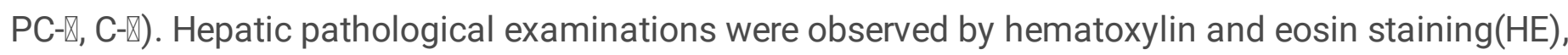
Masson staining and picric acid-Sirius red staining. The protein expression of TGF- $\beta 1$ and Smad3 were detected by immunohistochemistry. The level of mRNA expression of TGF- $\beta 1$ and Smad3 were detected by RT-PCR.

\section{Results}

Compared with the control group, serum levels of liver function indexes(TBA, TBil, DBil, ALT, AST, ALP, GGT) and liver fibrosis indexes(HA, LN, PC- $\mathrm{Q}, \mathrm{C}-\mathrm{-})$ in the model group were significantly increased $(\mathrm{P}<$ 0.01).Similarly, compared with control group, in the model group, HE staining showed that hepatocyte swelling, hepatocellular necrosis and destroyed hepatic lobular structure; Masson staining showed that a large amount of blue collagen fibers deposited in the liver tissue, forming fibrous septa and pseudolobule; Picric acid-Sirius red staining showed that type $\nabla$ collagen and type $\nabla$ collagen in liver tissue increased significantly, the total area of collagen increased significantly. The protein expression of TGF- $\beta 1$ and Smad3 were significantly up-regulated; the mRNA expression of TGF- $\beta 1$ and Smad3 was significantly upregulated. Compared with the model group, the above indexes in treatment group were significantly decreased $(P<0.01)$.

\section{Conclusions}


Babao Dan(BBD) has significant therapeutical effects on intrahepatic cholestasis liver disease by inhibiting TGF- $\beta$ /Smad signaling pathway.

\section{Introduction}

Cholestasis is a pathological condition characterized by obstruction of bile formation, secretion, and excretion caused by various causes inside and outside the liver ${ }^{[1]}$. Bile cannot be discharged normally into the duodenum, but instead enters the blood .Cholestatic liver disease is a global health problem, mainly refers to a variety of liver diseases with cholestasis as the main manifestation ${ }^{[2]}$. Liver diseases causing cholestasis are numerous and complex, mainly divided into intrahepatic cholestatic liver disease and extrahepatic cholestatic liver disease. Cholestasis is not only the main manifestation of various cholestatic liver diseases, but also causes further exacerbation of the disease, leading to the development of chronic liver disease and eventually cirrhosis ${ }^{[3]}$.The pathogenesis of cholestatic liver disease is not yet clear, and is related to various factors, including oxidative stress, cytokines (interleukin 6 (IL-6), tumor necrosis factor a (TNF-a), transforming growth factor $\beta$ (TGF- $\beta$ ), etc.), intestinal flora ${ }^{[4,5]}$, bile acid metabolism ${ }^{[1,5-7]}$, etc. .At present, a large number of studies have explored the pathogenesis of cholestatic liver disease, but no clear results have been obtained. By establishing a mouse model of MDR2 gene knockout cholestasis, Shearn CT et al. ${ }^{[2]}$ explored that cholestasis promotes liver inflammation, cytokines release, oxidative stress and protein carbonylation involvement leading to liver fibrosis and cirrhosis by inhibiting antioxidant effect.

Chronic cholestatic liver disease can eventually lead to liver fibrosis, cirrhosis and even liver cancer. Studies have confirmed that the activation of hepatic stellate cells(HSCs) is the key to liver fibrosis. Activation of hepatic stellate cells(HSCs) is affected by a variety of inflammatory cytokines ${ }^{\left[{ }^{[8]}\right.}$. TGF- $\beta$ is considered to be the strongest pro-fibrosis factor that can activate hepatic stellate cells and promote liver fibrosis. TGF- $\beta$ plays a pro-fibrosis role mainly through the TGF/Smad signaling pathway ${ }^{[9]}$. Smads protein is the only known intracellular kinase substrate of TGF- $\beta$ receptor, and is the key downstream protein of TGF- $\beta$ signaling transmembrane into the nucleus, regulating the transcription of nuclear target genes, thereby exerts the biological effects of TGF- $\beta^{[8-10]}$.

Chinese medicine plays an important role in the treatment of cholestasis, among which BBD is the most important one. As a traditional Chinese medicine, BBD originated in the Ming Dynasty. Its ingredients include bezoar, snake bile, antelope horn, pearl, notoginseng and musk, etc. It has the functions of clearing away dampness and heat, promoting blood circulation and detoxification, withdrawing yellow and relieving pain ${ }^{[5,11,12]}$. BBD has good efficacy in the treatment of viral hepatitis, liver cirrhosis, cholestatic liver disease and biliary tract disease ${ }^{[11,13-16]}$. Liang $L$ et al. ${ }^{[12]}$ demonstrated that BBD significantly inhibited LPS-induced activation of HSCs through TLR4/NF-KB pathway. In addition, BBD is

widely used for other diseases, such as tumor disease ${ }^{[17-19]}$, urinary system disease and so on. Traditional Chinese medicine plays a great role in the treatment of COVID-19. Previous studies ${ }^{[20]}$ have shown that BBD can improve the symptoms of COVID-19 combined with acute lung injury by controlling 
excessive inflammation and inhibiting the release of a large number of inflammatory cytokines by inflammatory cells. Similarly, the study suggests that octadine may have positive effects on cancer treatment. Liu et al. demonstrated through a variety of experimental methods that octadane inhibited the migration and invasion of gastric cancer cell lines by inhibiting TGF- $\beta$-induced epithelial mesenchymal transformation (EMT) and inactivating the TGF- $\beta /$ Smad signaling pathway ${ }^{[17]}$. Meanwhile, the study of Wang $Q$ et al. ${ }^{[18]}$ confirmed that BBD inhibited the growth of tumor cells by inducing autophagy through down-regulating PI3K/ATK/mTOR signaling pathway in lung cancer. However, there are few studies on the mechanisms of BBD in the treatment of cholestatic liver diseases, and there is still no clear conclusion.

At present, the mechanism of BBD in the treatment of cholestatic liver diseases, liver fibrosis and cirrhosis is still not clear. This study mainly observed the protective effect of BBD on a-naphthalene isothiocyanate (ANTI) -induced fibrosis in mice with cholestatic liver disease and explored its mechanisms, so as to provide more research evidences for the clinical application of BBD in the treatment of cholestatic liver diseases.

\section{Materials And Methods}

\section{Materials}

\subsection{Animals and groups}

15 healthy male SPF mice, Kunming species, body weight 20-25g, 28-33 days. Purchased from Guangdong Medical Laboratory Animal Center. Permit number SCXK (Yue) 2018-0002. Fifteen mice were randomly divided into 3 groups: control group, model group, and treatment group, with 5 mice in each group. It was raised under the SPF condition of the Animal Experimental Center of Zhujiang Hospital of Southern Medical University. Keep breeding environment illuminated for 12 hours, and keep the temperature of environment at $25^{\circ} \mathrm{C}$ and dry, every rat eats and drinks freely. All experimental procedures were conducted in strict accordance with the experimental guidelines of Animal Experimental Center of Zhujiang Hospital of Southern Medical University, and in accordance with the relevant provisions of animal protection, animal welfare and ethical principles.

\subsection{Drug}

BBD Capsules were purchased from Xiamen Chinese Pharmaceutical Factory Co., Ltd., Batch No. Z109440006, specification 0.3g/capsule. Take 0.6g BBD and then dilute it in $10 \mathrm{~mL}$ normal saline, make suspension by mixing, finally store it in refrigerator at $4^{\circ} \mathrm{C}$ for later use. In this study, a-Naphthyl isothiocyanate(ANIT) was used as the model drug for cholestatic liver disease. A variety of modeling methods and drugs for cholestatic liver diseases were investigated. ANIT is the most commonly used drug and can quickly form the model of acute cholestatic liver diseases ${ }^{[21]}$. ANIT was purchased from American sigma company. Precisely weigh ANIT $200 \mathrm{mg}$, then dissolve it in $20 \mathrm{~mL}$ olive oil to make oil solution with the concentration of $10 \mathrm{mg} / \mathrm{mL}$ before using. 


\subsection{Instruments and reagents}

Electronic balance(Mettler Toledo (Shanghai) Co., Ltd.), ultrasonic cell pulverizer (Ningbo Xinzhi Bio), table-top high-speed refrigerated centrifuge (Ningbo Xinzhi Bio), automatic grinder (Jiangyan Tianli Medical Instrument Factory Co., Ltd.), automatic biochemical analyzer (Shenzhen Leidu Life Technology), dehydrator (Wuhan Junjie Electronics Co., Ltd.), pathological slicer (Shanghai Leica Instrument Co., Ltd.), upright optics Microscope (Nikon, Japan), fluorescent quantitative PCR (ABI), ultra-micro spectrophotometer (Thermo), centrifuge tube (Axygen Biosciences), imaging system (Nikon, Japan); scissors, tweezers, EP tube are offered by the Animal Experimental Center of the Southern Medical University Zhujiang Hospital.

\section{Method}

\subsection{Preparation of cholestatic cirrhosis rat models}

15 mice were adaptively fed for 24 hours, and then randomly divided into 3 groups: control group, model group, and treatment group. Mice in the model group and treatment group were gavaged with $1 \%$ ANIT olive oil solution at one time, the model of cholestasis was prepared at the dose of $100 \mathrm{mg} / \mathrm{Kg}$. The control group was gavaged with the same amount of olive oil at the same time point. Before and after modeling, fast and drink water for 6 hours to ensure full absorption of the drug. The control group was given normal saline gavage once a day, the model group was gavaged with normal saline once a day, and the treatment group was gavaged BBD normal saline suspension once a day at a dose of $1.2 \mathrm{~g} / \mathrm{Kg}$ (twice the human equivalent dose) for 10 days. After 10 days of administration, blood samples and liver tissues of each group were collected after anesthesia.

\subsection{Materials and testing indicators}

\subsubsection{Observe the general condition of the mice, dietary activities, mental state, and condition of death.}

\subsubsection{Mouse serum biochemical test}

On day 10 of the experiment, all animals were fasted for 12 hours after administration, and then anesthetized by intraperitoneal injection of $250 \mathrm{mg} / \mathrm{kg} 6 \%$ chloral hydrate. After the eyes were removed, blood was collected, $1.5 \mathrm{ml}$ arterial blood was extracted and placed in the procoagulant tube. The blood was separated by centrifuge at $4000 \mathrm{r} / \mathrm{min}$ for $10 \mathrm{~min}$, and serum was collected for examination. All the serum biochemical indicators in this experiment such as liver function indexes(TBA, TBil, DBil, ALT, AST,

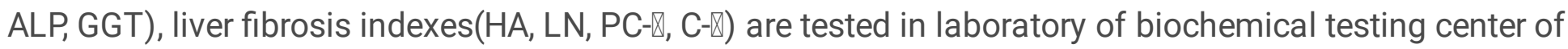
Zhujiang Hospital of Southern Medical University.

\subsubsection{Pathological observation of liver tissue}

Fresh liver tissue was collected, and the same part of liver was fixed in $10 \%$ neutral formalin buffer solution for HE staining, Masson staining and Picric acid-Sirius red staining. Liver tissue inflammation 
and fibrous collagen deposition were observed. Image-Pro Plus6.0 software was used to calculate the collagen fiber area. The preparation of liver pathological sections and HE staining were completed by the laboratory of Department of Pathology, Zhujiang Hospital, Southern Medical University.

\subsubsection{The expression level of TGF- $\beta 1$ and Smad3 protein}

The expression level of TGF- $\beta 1$ and Smad3 protein was detected by immunohistochemical stain. Hematoxylin stains nucleus to blue, DAB showed positive expression is claybank, under the observation at high magnification (400 times), randomly observe 5 overlapping fields of vision, the counts of cells in the every field should be over 300 , dyeing results should be compared with the proportion of positive cells and staining extent to make the semi-quantitative analysis of TGF- $\beta 1$, Smad3 protein expression. The result evaluation criteria: the staining color of cytoplasm and cell membrane of positive cells is yellow or claybank, and the cells without staining were taken as negative cells. The image-pro Plus6.0 Image analysis software was used for semi-quantitative analysis of the positive expression regions.

\subsubsection{Detection of TGF- $\beta 1$ and Smad3 mRNA expression levels by PT-PCR}

Accurately weigh liver tissue $10 \mathrm{mg}$ per mouse, then grind after adding with $250 \mu$ lysate. Follow-up operations are performed according to the instructions on the total RNA extraction kit. The proposed RNA concentration was detected by ultraviolet spectrophotometry, and 2.0 $\mu$ l of total RNA was added into each tube for reverse transcription. Add the reagent according to the instructions, place it in the PCR apparatus, and incubate at $65^{\circ} \mathrm{C}$ for $5 \mathrm{~min}$ and $42^{\circ} \mathrm{C}$ for $60 \mathrm{~min}$. The internal reference and primer amplification conditions were as follows: pre-denaturation at $94^{\circ} \mathrm{C}$ for $10 \mathrm{~min}$, denaturation at $95^{\circ} \mathrm{C}$ for $15 \mathrm{~s}$, annealing at $60^{\circ} \mathrm{C}$ for $60 \mathrm{~s}$, and extension at $70^{\circ} \mathrm{C}$ for $1 \mathrm{~min}$, a total of 40 cycles.(see table 1 )

\subsection{Statistical Methods}

The experimental data was analyzed by SPSS 17.0 statistical analysis software, results were expressed with mean \pm standard deviation $(x \pm s)$. For the data conforming to homogeneity of variance, group $t$ test was used for pair comparison, and one-way analysis of variance was used for comparison of the mean between multiple groups. $P<0.01$ was considered statistically significant.

\section{Results}

\section{BBD improved the general condition of mice of the treatment group compared with that of mice of the model group.}

As for the control group, the mice were in good mental condition with normal activity ,stable body weight, bright fur, light red limbs and mouth corners, slightly yellow urine, and moderate stool humidity.

Compared with control group, the general condition of mice in the model and treatment group was worse. The mental state was worse with declined activity level, slow movement and weight loss. The Fur on head and back was lost and dull. The color of limbs, mouth corners and tail circumference became yellow, the color of urine became yellow and the volume decreased, stool became dry. The mice became 
emotional, defensive, and had aggressivity and aggressive behavior. After administration, compared with the model group, the general condition of mice in the treatment group improved. As the time of administration increase, the mental state ,activity level, fur and urine improved. During the administration period, there were no obvious adverse reactions occurred in each model group.

\section{BBD made the liver function indexes decreased and improved the liver function.}

Compared with the control group, liver functional indexes (TBA, TBil, DBil, ALT, AST, ALP, GGT) in the model group were significantly increased $(P<0.01)$. Compared with the model group, liver functional indexes(TBA, TBil, DBil, ALT, AST, ALP, and GGT) in the treatment group were significantly decreased $(P<0.01)$, the differences among all groups were statistically significant.(See Table 2$)$

Compared with the control group, the liver fibrosis indexes (LN, HA, PC- $₫$ and C- $)$ in the model group and the treatment significantly increased $(P<0.01)$. Compared with the model group, the liver fibrosis indexes $(\mathrm{LN}, \mathrm{HA}, \mathrm{PC}-\mathbb{\nabla}$ and $\mathrm{C}-\mathrm{-})$ in the treatment group significantly decreased $(\mathrm{P}<0.01)$, and the differences between the groups were statistically significant. (See Table 3)

\section{BBD improved liver tissue structure and inhibited collagen synthesis in the liver.}

\subsection{HE staining}

In the control group, liver cells were neatly arranged and cord-like radiated without necrosis or degeneration. The structure of liver lobules was clear and complete, the structure of portal area was normal, and the epithelial cells of bile duct were neatly arranged without blockage. No abnormal hyperplasia of connective tissue was observed in the central vein and portal area, and no inflammatory cell infiltration was observed. In the model group, mice liver cells cord arrangement was disorder and irregular, liver cell became swelling, and had fat necrosis, balloon-like degeneration, large area of necrosis, connective tissue in the necrosis area significantly increased, forming fibrous septum, which destroyed liver lobular structure and then gradually became false lobules, finally developed to liver fibrosis. Meanwhile, it could be found that the significant hyperplasia of bile duct epithelial cells, the obstruction of bile duct and the infiltration of a large number of inflammatory cells in the portal area. Compared with model group, the arrangement and hierarchy of liver cells in the treatment group were more regular, the cell size was more uniform, and the morphology and structure were clearer. The edema of liver cells, fatty necrosis of hepatocytes, necrotic area of liver, obstruction of bile duct and inflammatory cell infiltration in portal area were significantly reduced. The liver injury was improved obviously, and the tissue structure and cell morphology were close to normal condition compared with model group. (see Figure1)

\subsection{Masson staining}

A small amount of collagen fibers were seen in the wall of the control group, which is in the normal range. Compared with the control group, a large number of bright blue collagen fibers deposited in the liver tissue of the model group, and the staining was deeper. More collagen fibers were found in the liver 
tissue, fiber spacing varied in width, and pseudo-lobules were formed. The change of collagen fibers in the treatment group were between the control group and the model group. (see Figure 2)

\subsection{Picric acid-Sirius red staining( $($ and III collagen fibers observed by polarized light microscopy)}

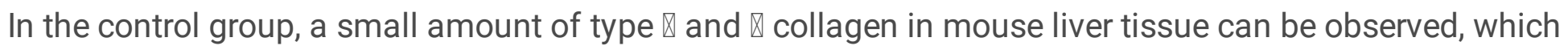
were distributing in the portal area, blood vessel wall and bile duct wall. By contrast, in the model group, the type $\otimes$ collagen in liver tissue increased significantly, which was distributing in the space around the hepatic sinus and the lobular vein and partially extending into the lobules, then forming fibrous septa between the leaves. There were a large number of orange-red crude fibers in the portal area with strong refraction. Type $\nabla$ collagen is increased and scattered around type $\otimes$ collagen. Compared with the model group, in the treatment group, collagen fibers in liver tissue are mainly composed of green type $₫$ collagen which were radiated in filaments and showed weak birefringence. The green type $₫$ collagen mainly distributed in the portal area and small blood vessel wall, meanwhile, it also can be observed around the hepatic sinus. Type $\otimes$ collagen was accompanied by type $\otimes$ collagen which showed red or yellow coarse fibrousa and strong birefringence. (See Figure 3)

\subsection{Collagen fiber area analysis}

Collagen fiber area analysis showed that the collagen area of the model group increased significantly compared with the control group, and the differences was statistically significant $(P<0.01)$.Compared with the model group, the collagen area of the treatment group significantly decreased, and the differences was statistically significant $(P<0.01)$. There was no significant differences of the area of fibrosis between Masson staining and picric acid-Sirius red staining between the same group $(P>0.01)$. (See Table 4)

\section{BBD inhibited expression levels of TGF- $\beta 1$ and Smad3 proteins in mouse liver tissues}

\subsection{TGF- $\beta 1$ expression}

A few brownish yellow positive granules in liver tissue of control group were observed. No positive expression was found in hepatocytes and interstitial cells, and a small amount of positive expression was found in hepatic sinus space. A large number of colored particles can be seen in the model group. In the fibrosis portal areas and increased fibrous tissue areas, it showed mainly that a large number of positive staining and cytoplasmic expression were observed in interstitial cells, cytoplasm of inflammatory cells, spaces between hepatic sinuses and wall of central venous vessels. It can be seen that TGF- $\beta 1$ is mostly derived from mesenchymal cells. The expression of TGF- $\beta 1$ protein in the treatment group was lower than that of the model group, the number of positive cells was significantly reduced, and the degree of staining was significantly reduced, the TGF- $\beta 1$ protein mainly expressed on the interstitial cell membrane and in the cytoplasm. Compared with the normal group, the expression of TGF- $\beta 1$ protein in the model group and the treatment group increased significantly, and the differences were statistically significant $(P<0.01)$. Compared with the model group, the expression of TGF- $\beta 1$ protein in the treatment 
group was decreased obviously, and the differences were statistically significant $(P<0.01)$. (See Figure 4, Table 5)

\subsection{Smad3 expression}

There was no positive expression of hepatocytes in the control group, and a small amount of chromogenic particles were observed in the portal areas. Compared with control group, a large number of positive chromogenic particles were observed in the model group, mainly locating in the portal areas, hepatic sinus spaces, fibrous areas, and around the blood vessel wall, and the expression of Smad3 protein was mainly cytoplasmic expression. The positive expression of the treatment group was less than that of the model group, and the positive particles were significantly reduced. Compared with the control group, the expression of Smad3 protein positive particles in the model group and treatment group increased significantly, and the differences were statistically significant $(P<0.01)$.Compared with the model group, the expression of Smad3 protein positive particles in the treatment group was decreased obviously, and the differences was statistically significant $(P<0.01)$. (See Figure 5, Table 5)

\section{Discussion}

Cholestasis liver disease is a kind of the liver diseases with cholestasis as the main performance, including a variety of diseases, such as primary biliary cholangitis (PBC) and primary sclerosing cholangitis (PSC),etc., is characterized in bile synthesis, secretion and excretion dysfunction, leading to chronic bile acids accumulation within the liver and causing liver damage ${ }^{[22]}$. Excessive accumulation of bile acids and their metabolites in the liver is considered to be the main cause of liver injury in cholestatic liver diseases ${ }^{[2,21,23]}$. At present, the first-line treatment drug for cholestatic liver disease is ursodeoxycholic acid, but unfortunately, many patients are not sensitive to ursodeoxycholic acid, and the efficacy is poor ${ }^{[1,24]}$. Meanwhile, there are not many drugs for cholestatic liver disease, and the curative effect varies greatly ${ }^{[6]}$. As a traditional Chinese medicine, BBD is widely used in many kinds of liver diseases, as well as cholestatic liver diseases. It is widely used in China with good curative effect ${ }^{[11,15,16 \text {, }}$ 18]. The mechanism of BBD in the treatment of cholestatic liver diseases is still unclear. However, it has been suggested that BBD can exert anti-fibrosis effect through inhibiting TGF- $\beta$ /Smad signaling pathway, and inhibit tumor cell proliferation and metastasis in NSCLC ${ }^{[14,17,18]}$. The purpose of this study was to observe the protective effect of BBD on fibrosis in mice with cholestatic liver disease induced by anaphthalene isothiocyanate (ANTI) and explore its mechanism. The results of this study suggested that BBD has a protective effect on liver injury and improves liver function in cholestatic liver disease, which may be achieved by inhibiting the TGF- $\beta$ /Smad signaling pathway.

In this study, the mouse model of cholestasis liver fibrosis was prepared by ANIT gavage method ${ }^{[21]}$. After preparation, the general state of mice in each group was observed, the serum biochemical indexes of mice were detected, and the liver of mice was stained with HE. The results showed that the general condition of mice in the model group was significantly lower than those in the control group, with reduced activity, jaundice and sparse fur. Compared with the control group, the liver function indexes of the model 
group were significantly increased, the liver function was significantly impaired, and the liver fibrosis indexes were significantly increased. At the same time, HE staining of liver tissue of mice in model group showed hepatocyte swelling, large area necrosis, pseudolobule formation, bile duct obstruction and inflammatory cell infiltration. The results were consistent with previous studies. Compared with the model group, the general condition of mice in the BBD treatment group was significantly improved with increased activity and reduced jaundice. Compared with the model group, serum biochemical indexes, liver fibrosis indexes and liver function indexes of mice in the treatment group were significantly decreased. HE staining of liver tissue of mice in the treatment group showed that the edema of liver cells was reduced, liver injury was significantly improved, and tissue structure and cell morphology were close to normal. The experimental results confirmed that BBD had a protective effect on liver injury caused by cholestatic liver disease, and the liver function was improved obviously.

In addition, in order to further quantitatively verify the protective effect of BBD on liver injury caused by cholestasis liver disease, we performed Masson staining and picric acid-sirius red staining on liver tissue of mice in each group, and quantitatively analyzed the distribution area of type I and $\nabla$ collagen. Masson staining results showed that the collagen area of the model group was significantly increased, while the collagen area of the treatment group was significantly decreased. The results of picric acid-sirius red staining were consistent with those of Masson staining. The collagen distribution area of the model group increased significantly, while those of the treatment group decreased. The results of the collagen distribution area calculated by computer software showed that there were significant differences between the model group and the treatment group. The experimental results showed that BBD could significantly improve the collagen hyperplasia and liver fibrosis caused by cholestasis liver injury.

At present, there have been a large number of studies related to the pathogenesis of cholestatic liver disease, which is very complex. Studies ${ }^{[8,10,25]}$ have shown that TGF- $\beta$ plays a crucial role in liver fibrosis, activating hepatic stellate cells, activating downstream Smad expression, and promoting the occurrence and development of liver fibrosis. In order to further explore the mechanism of BBD in the treatment of cholestatic liver disease, we analyzed the protein expression levels of TGF- $\beta$ and Smad in liver by immunohistochemistry, and detected the mRNA expression levels of TGF- $\beta$ and Smad by PT-PCR. The results showed that the expressions of TGF- $\beta$, Smad and mRNA were significantly increased in the model group and significantly decreased in the treatment group compared with the model group. The results have shown that cholestatic liver disease may cause liver damage through activation of the TGF- $\beta /$ Smad signaling pathway. The expression of TGF- $\beta$ and Smad decreased significantly after BBD treatment, suggesting that BBD may improve liver injury and fibrosis in cholestatic liver disease by inhibiting TGF$\beta /$ Smad signaling pathway, which is consistent with the results of previous studies.

In conclusion, we suggest that BBD has a protective effect on cholestatic liver disease caused by anaphthalene isothiocyanate (ANTI), which can improve liver injury and liver function, and this protective effect may be realized by inhibiting TGF- $\beta /$ Smad signaling pathway. Because many patients have poor response to ursodeoxycholic acid treatment, the efficacy of other therapeutic drugs still needs further study, and BBD has broad application space. Due to the differences among species, there are still many 
shortcomings in this study. The modeling drugs used in this study were mainly presented with acute cholestasis, and their response to chronic cholestasis was unclear. In this study, mice could not completely replace the human condition, which was more complicated, and the therapeutic effect was not clear yet. Further clinical studies were needed to confirm the therapeutic effect of BBD. This study on the therapeutic mechanism of BBD, it was still relatively simple, while the pathogenesis of cholestatic liver disease is very complex, which is related to a variety of signal pathways, molecules and metabolites. The mechanism still needs to be further studied to explore its correlation. Therefore, the research on cholestatic liver disease still needs to be further in-depth. According to the current research results, we believe that BBD has a good therapeutic effect on cholestatic liver disease and has a broad application space and value.

\section{Conclusion}

The results of our study showed that the general condition of mice in the Babao Dan(BBD) treatment group was significantly improved, and the fibrosis status of liver tissue was significantly improved. The PT-PCR results showed that the mRNA expression of TGF- $\beta$ and Smad in the Babao Dan(BBD) treatment group was significantly reduced. Therefore, we suggest thatBabao Dan(BBD) has significant therapeutical effects on intrahepatic cholestasis liver disease by inhibiting TGF- $\beta /$ Smad signaling pathway.

\section{Abbreviations}

BBD

Babao Dan; ALT:alanine aminotransferase; AST:aspartate aminotransferase; ANIT:a-Naphthyl isothiocyanate; TBA:total bile acid; TBil:total bilirubin; DBil:direct bilirubin; ALP:alkaline phosphatase;

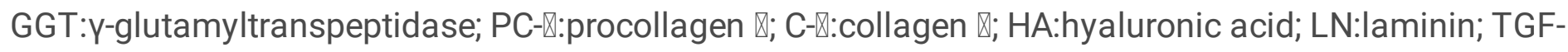
$\beta$ :transforming growth factor $\beta$; RT-PCR:reverse transcription-polymerase chain reaction.

\section{Declarations}

\section{Acknowledgements}

Thanks to all colleagues who helped me with my experiment.

\section{Authors' contributions}

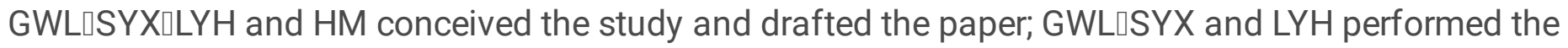

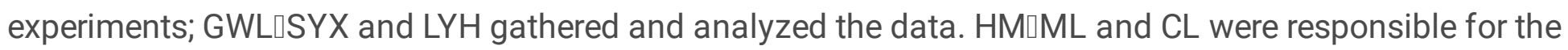
revision and improvement of the article. All authors read and approved the final manuscript

\section{Availability of data and materials}


Please contact the corresponding author for data requests.

\section{Ethics approval and consent to participate}

All mouse experiments and procedures were approved by the Animal Ethics Committee of Zhujiang hospital of Southern Medical University, Guangzhou, China, and all experiments were performed in accordance with the approved guidelines and regulations.

\section{Consent for publication}

All authors reviewed and approved the final manuscript. All authors sup- ported publication of this manuscript.

\section{Competing interests}

The authors declare that they have no competing interests.

\section{Funding}

Not applicable

\section{Author details}

*Correspondence: 1109663087@qq.com

${ }^{1}$ Gastroenterology department, Zhujiang Hospital, Southern Medical University, 253 Middle Industrial Avenue, Guangzhou 510300, China.

\section{References}

1. Mazzetti M, Marconi G, Mancinelli M, Benedetti A, Marzioni M, Maroni L: The Management of Cholestatic Liver Diseases: Current Therapies and Emerging New Possibilities. Journal of clinical medicine 2021, 10(8).

2. Shearn CT, Fennimore B, Orlicky DJ, Gao YR, Saba LM, Battista KD, Aivazidis S, Assiri M, Harris PS, Michel $C$ et al: Cholestatic liver disease results increased production of reactive aldehydes and an atypical periportal hepatic antioxidant response. Free radical biology \& medicine 2019, 143:101-114.

3. Azad Al, Krishnan A, Troop L, Li Y, Katsumi T, Pavelko K, Kostallari E, Guicciardi ME, Gores GJ: Targeted Apoptosis of Ductular Reactive Cells Reduces Hepatic Fibrosis in a Mouse Model of Cholestasis. Hepatology (Baltimore, Md) 2020, 72(3):1013-1028.

4. Kummen M, Hov JR: The gut microbial influence on cholestatic liver disease. Liver international : official journal of the International Association for the Study of the Liver 2019, 39(7):1186-1196.

5. Li Y, Tang R, Leung PSC, Gershwin ME, Ma X: Bile acids and intestinal microbiota in autoimmune cholestatic liver diseases. Autoimmunity reviews 2017, 16(9):885-896. 
6. Ovadia C, Sajous J, Seed PT, Patel K, Williamson NJ, Attilakos G, Azzaroli F, Bacq Y, Batsry L, Broom $\mathrm{K}$ et al: Ursodeoxycholic acid in intrahepatic cholestasis of pregnancy: a systematic review and individual participant data meta-analysis. The lancet Gastroenterology \& hepatology 2021.

7. Olaf T, Christian J, Robert S, Erhard UF, Mads I, Sabine K, Cristina O, P SC, Stefan Z, Wenyi G et al: Bile microbiome varies by etiology of cholestatic liver disease. \% J Liver transplantation : official publication of the American Association for the Study of Liver Diseases and the International Liver Transplantation Society. 2020.

8. Mu M, Zuo S, Wu RM, Deng KS, Lu S, Zhu JJ, Zou GL, Yang J, Cheng ML, Zhao XK: Ferulic acid attenuates liver fibrosis and hepatic stellate cell activation via inhibition of TGF- $\beta /$ Smad signaling pathway. Drug design, development and therapy 2018, 12:4107-4115.

9. Kang R, Tian W, Cao W, Sun Y, Zhang HN, Feng YD, Li C, Li ZZ, Li XQ: Ligustroflavone ameliorates $\mathrm{CCI}(4)$-induced liver fibrosis through down-regulating the TGF- $\beta / S m a d$ signaling pathway. Chinese journal of natural medicines 2021, 19(3):170-180.

10. Liu J, Kong D, Qiu J, Xie Y, Lu Z, Zhou C, Liu X, Zhang R, Wang Y: Praziquantel ameliorates CCI(4) induced liver fibrosis in mice by inhibiting TGF- $\beta /$ Smad signalling via up-regulating Smad7 in hepatic stellate cells. British journal of pharmacology 2019, 176(24):4666-4680.

11. Lu L, Wu C, Lu BJ, Xie D, Wang Z, Bahaji Azami NL, An YT, Wang HJ, Ye G, Sun MY: BabaoDan cures hepatic encephalopathy by decreasing ammonia levels and alleviating inflammation in rats. Journal of ethnopharmacology 2020, 249:112301.

12. Liang L, Yang X, Yu Y, Li X, Wu Y, Shi R, Jiang J, Gao L, Ye F, Zhao Q et al: Babao Dan attenuates hepatic fibrosis by inhibiting hepatic stellate cells activation and proliferation via TLR4 signaling pathway. Oncotarget 2016, 7(50):82554-82566.

13. Bingjie L, Chao W, Bahaji ANL, Dong X, Changqing Z, Wan X, Dengcheng $H, X i C$, Runfei S, Jingru $S$ et al: Babao Dan improves neurocognitive function by inhibiting inflammation in clinical minimal hepatic encephalopathy. Biomedicine \& pharmacotherapy = Biomedecine \& pharmacotherapie 2020, 135.

14. Yu Y, Tian ZQ, Liang L, Yang X, Sheng DD, Zeng JX, Li XY, Shi RY, Han ZP, Wei LX: Babao Dan attenuates acute ethanol-induced liver injury via Nrf2 activation and autophagy. Cell \& bioscience 2019, 9:80.

15. Sheng D, Zhao S, Gao L, Zheng H, Liu W, Hou J, Jin Y, Ye F, Zhao Q, Li R et al: BabaoDan attenuates high-fat diet-induced non-alcoholic fatty liver disease via activation of AMPK signaling Cell \& bioscience 2019, 9(1).

16. Gong W, Liu L, Li M, Wang L, Zhang M, Luo Z, Sridhar S, Woo PCY, Wang L: Evaluation of antiviral efficacy of Chinese traditional medicine Babao Dan in rabbits infected with hepatitis $E$ virus. The Journal of general virology 2018, 99(8):1036-1043.

17. Liu J, Chen Y, Cao Z, Guan B, Peng J, Chen Y, Zhan Z, Sferra TJ, Sankararaman S, Lin J: Babao Dan inhibits the migration and invasion of gastric cancer cells by suppressing epithelial-mesenchymal 
transition through the TGF- $\beta /$ Smad pathway. The Journal of international medical research 2020 , 48(6):300060520925598.

18. Wang Q, Liu Z, Du K, Liang M, Zhu X, Yu Z, Chen R, Qin L, Li Y, Zheng Y: Babaodan inhibits cell growth by inducing autophagy through the PI3K/AKT/mTOR pathway and enhances antitumor effects of cisplatin in NSCLC cells. American journal of translational research 2019, 11(8):52725283.

19. Song LB, Gao S, Zhang AQ, Qian X, Liu LM: Babaodan Capsule 0 combined with Qingyi Huaji Formula 0 in advanced pancreatic cancer-a feasibility study. Chinese journal of integrative medicine 2017, 23(12):937-942.

20. Qian J, Xu H, Lv D, Liu W, Chen E, Zhou Y, Wang Y, Ying K, Fan X: Babaodan controls excessive immune responses and may represent a cytokine-targeted agent suitable for COVID-19 treatment. Biomedicine \& pharmacotherapy = Biomedecine \& pharmacotherapie 2021, 139:111586.

21. Gijbels E, Pieters A, De Muynck K, Vinken M, Devisscher L: Rodent models of cholestatic liver disease: A practical guide for translational research. Liver international : official journal of the International Association for the Study of the Liver 2021, 41(4):656-682.

22. Jansen PL, Ghallab A, Vartak N, Reif R, Schaap FG, Hampe J, Hengstler JG: The ascending pathophysiology of cholestatic liver disease. Hepatology (Baltimore, Md) 2017, 65(2):722-738.

23. Panzitt K, Fickert P, Wagner M: Regulation of autophagy by bile acids and in cholestasis CholestoPHAGY or CholeSTOPagy. Biochimica et biophysica acta Molecular basis of disease 2021, 1867(2):166017.

24. Santiago P, Scheinberg AR, Levy C: Cholestatic liver diseases: new targets, new therapies. Therapeutic advances in gastroenterology 2018, 11:1756284818787400.

25. Narmada BC, Chia SM, Tucker-Kellogg L, Yu H: HGF regulates the activation of TGF- $\beta 1$ in rat hepatocytes and hepatic stellate cells. Journal of cellular physiology 2013, 228(2):393-401.

\section{Tables}

Due to technical limitations, table $1,4,5$ is only available as a download in the Supplemental Files section.

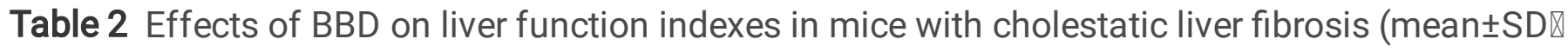




\begin{tabular}{|llllll|}
\hline & control group & model group & treatment group & $F$ & $P$ \\
\hline ALT(U/L) & $42.44 \pm 4.93$ & $400.33 \pm 65.49^{\mathrm{a}}$ & $144.16 \pm 38.66^{\mathrm{ab}}$ & 87.28 & 0.00 \\
\hline AST(U/L) & $171.09 \pm 22.30$ & $656.95 \pm 118.17^{\mathrm{a}}$ & $315.81 \pm 75.65^{\mathrm{ab}}$ & 46.24 & 0.00 \\
\hline ALP(U/L) & $158.82 \pm 39.18$ & $953.64 \pm 282.86^{\mathrm{a}}$ & $535.99 \pm 131.42^{\mathrm{ab}}$ & 23.99 & 0.00 \\
\hline GGT(U/L) & $29.58 \pm 3.49$ & $159.49 \pm 24.78^{\mathrm{a}}$ & $81.53 \pm 13.59^{\mathrm{ab}}$ & 79.12 & 0.00 \\
\hline TBIL(umol/L) & $6.31 \pm 0.91$ & $53.12 \pm 7.51^{\mathrm{a}}$ & $19.42 \pm 1.81^{\mathrm{ab}}$ & 144.56 & 0.00 \\
\hline DBIL(umol/L) & $4.54 \pm 0.86$ & $48.49 \pm 7.08^{\mathrm{a}}$ & $14.26 \pm 2.07^{\mathrm{ab}}$ & 144.77 & 0.00 \\
\hline TBA(umol/L) & $39.01 \pm 3.05$ & $339.47 \pm 167.73^{\mathrm{a}}$ & $238.28 \pm 103.87^{\mathrm{ab}}$ & 25.41 & 0.00 \\
\hline
\end{tabular}

Table 2: ${ }^{a}$ Compared with the data in the control group, the differences between the control group and the other groups have statistically significance $(P<0.01) .{ }^{b}$ Compared with the data in the model group, the differences of data have statistically significance $(P<0.01)$.

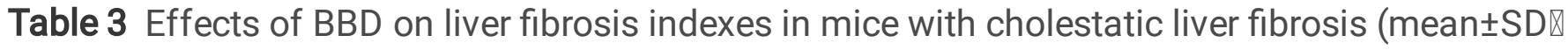

\begin{tabular}{|c|c|c|c|c|c|}
\hline & control group & model group & treatment group & $\mathrm{F}$ & $\mathrm{P}$ \\
\hline $\mathrm{HA}$ & $302.16 \pm 45.95$ & $644.21 \pm 71.69^{a}$ & $443.79 \pm 47.06^{a b}$ & 46.83 & 0.00 \\
\hline LN & $135.13 \pm 49.31$ & $417.55 \pm 37.76^{a}$ & $224.33 \pm 48.61^{a b}$ & 50.28 & 0.00 \\
\hline $\mathrm{PC}-\mathrm{\nabla}$ & $12.15 \pm 1.87$ & $24.94 \pm 2.02^{\mathrm{a}}$ & $16.15 \pm 1.03^{a b}$ & 73.83 & 0.00 \\
\hline$C-\square$ & $37.17 \pm 2.00$ & $74.98 \pm 4.34^{a}$ & $54.92 \pm 4.44^{\mathrm{ab}}$ & 125.89 & 0.00 \\
\hline
\end{tabular}

Table 3: a Compared with the data in the control group, the differences between the control group and the other groups have statistically significance $(\mathrm{P}<0.01) .{ }^{\mathrm{b}}$ Compared with the data in the model group, the differences of data have statistically significance $(P<0.01)$.

\section{Figures}




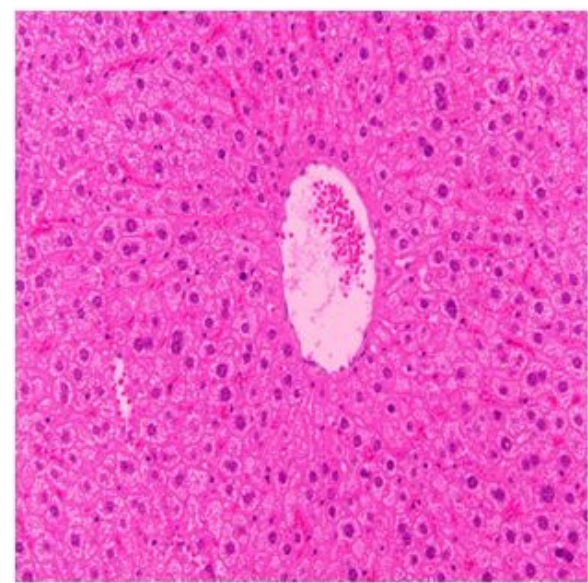

A. control group

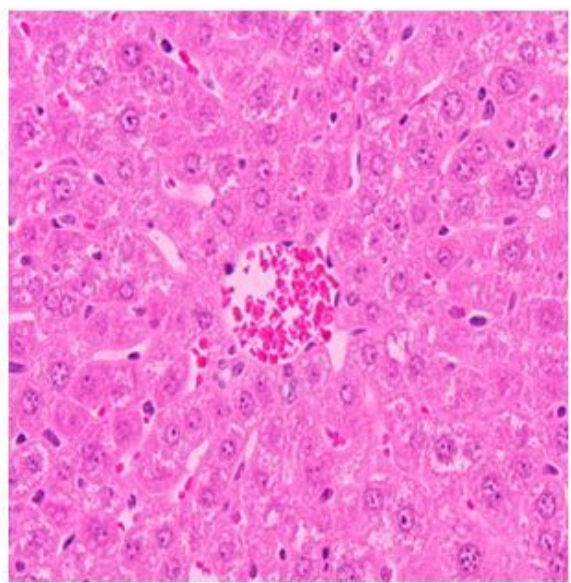

B. model group

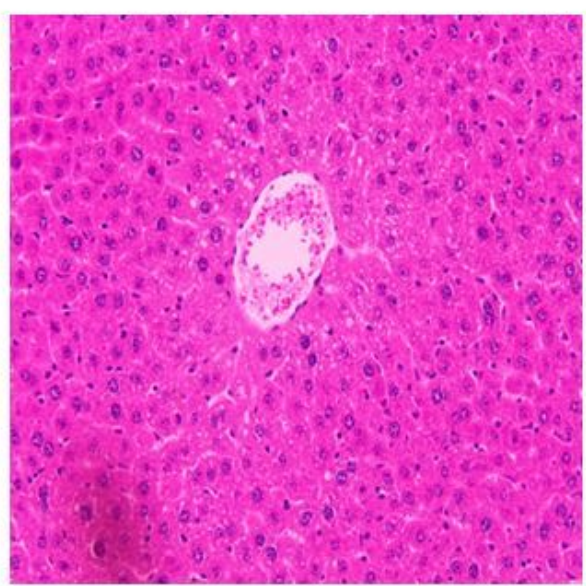

C. treatment group

\section{Figure 1}

Comparison of histopathology of liver tissue of each group(X40).

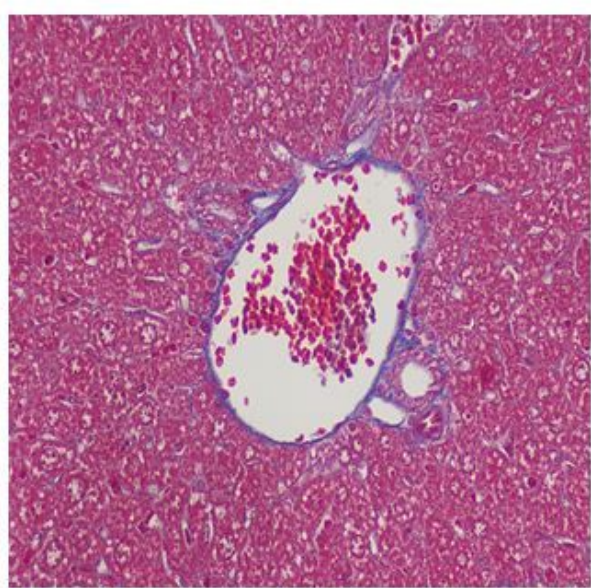

A. control group

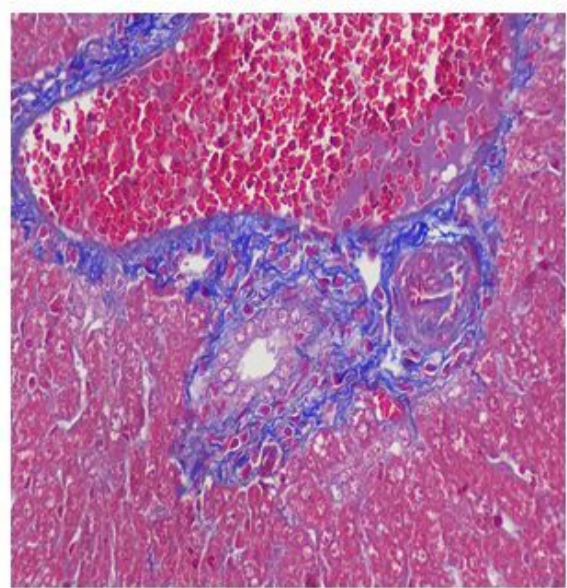

B. model group

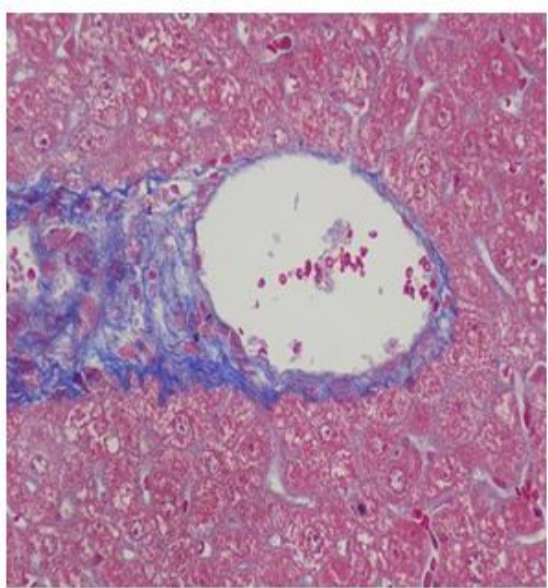

C. treatment group

Figure 2

Comparison of Masson staining of liver tissue of each group(X40). 


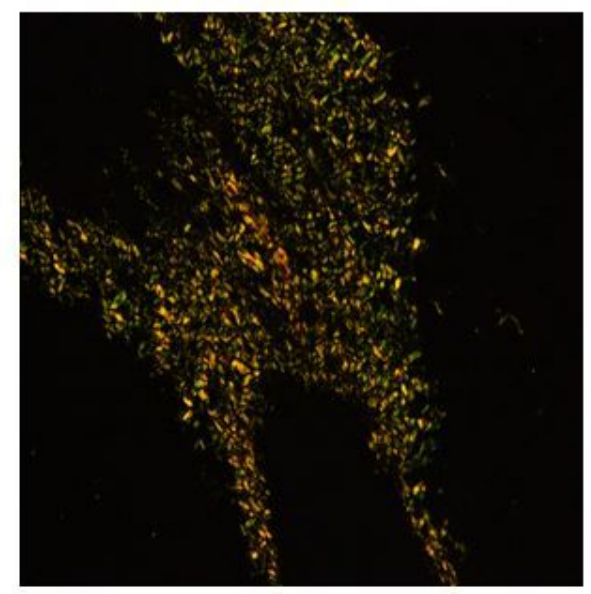

A. control group

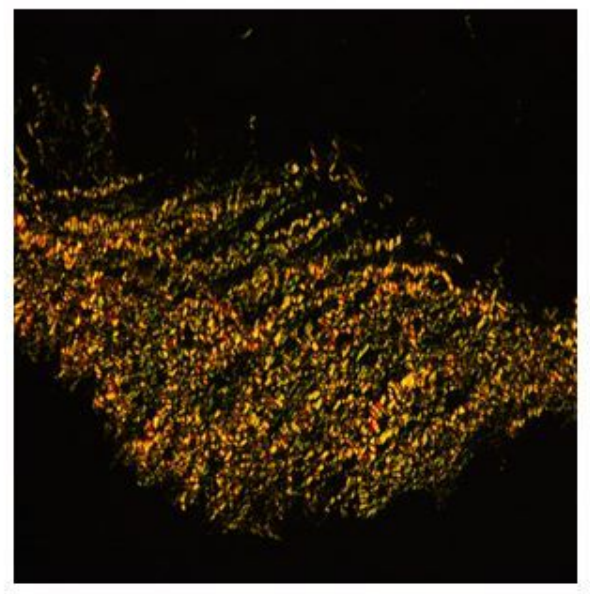

B. model group

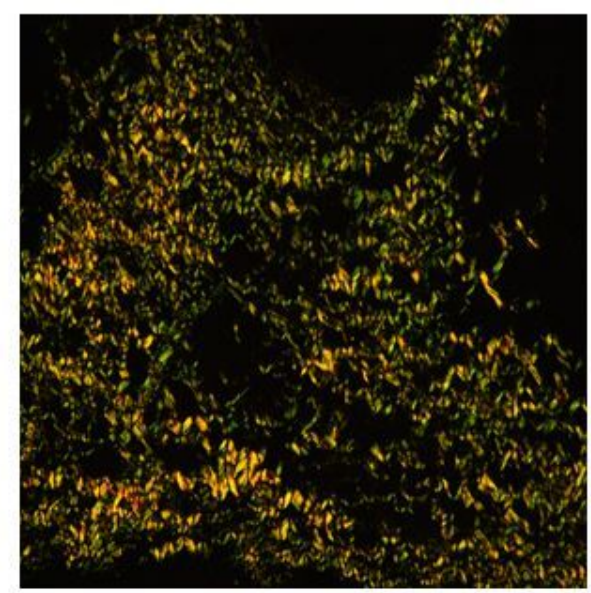

C. treatment group

\section{Figure 3}

Comparison of Picric acid - Sirius red staining of liver tissue of each group(X40).

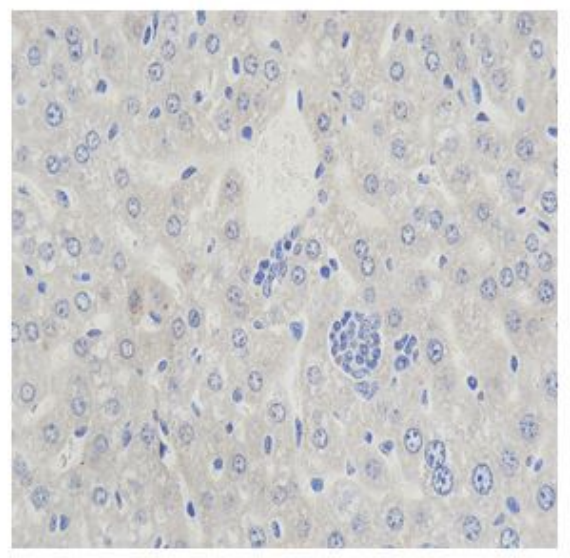

A. control group

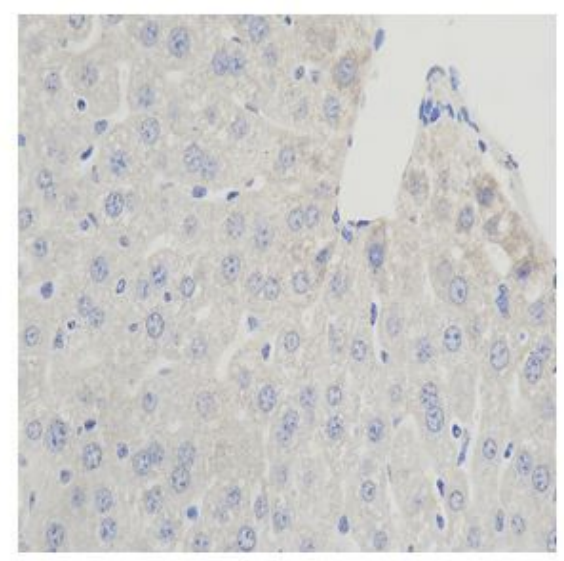

B. model group

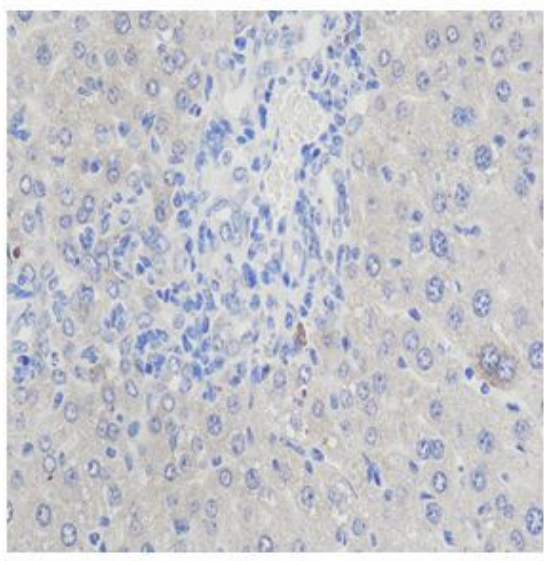

C. treatment group

Figure 4

Comparison of the protein of TGF- $\beta 1$ between normal group, model group and treatment group. 


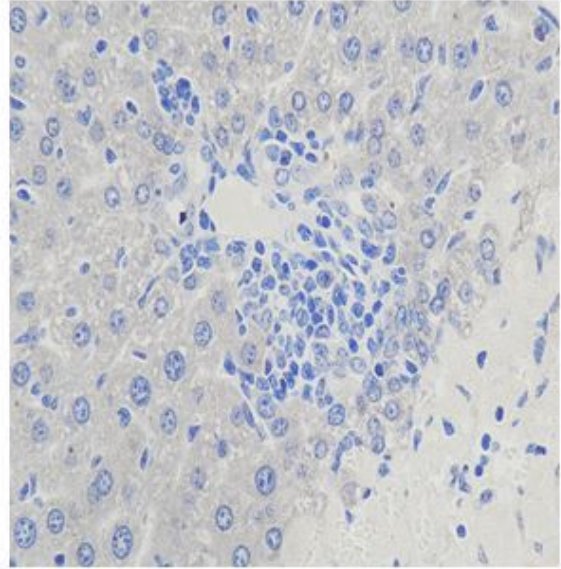

A. control group

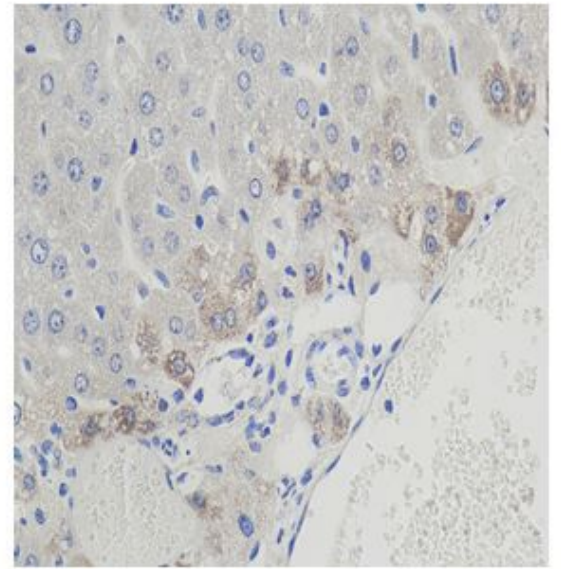

B. model group

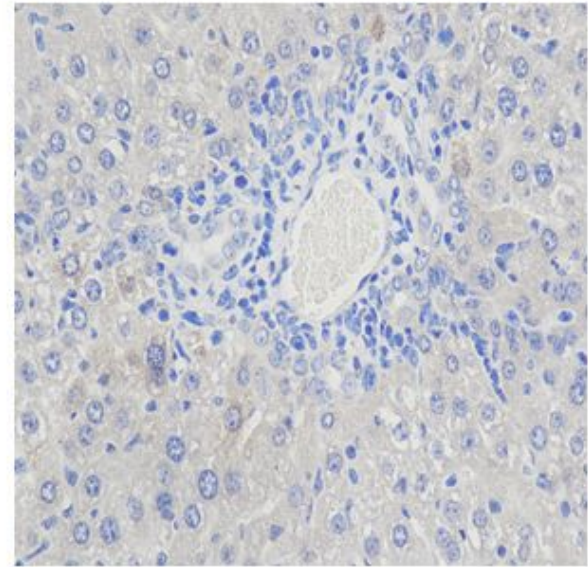

C. treatment group

Figure 5

Comparison of the protein of Smad3 between control group, model group and treatment group

\section{Supplementary Files}

This is a list of supplementary files associated with this preprint. Click to download.

- Table1.png

- Table4.png

- Table5.png 